\title{
Impacts of Network Constraints on Electricity Market Equilibrium
}

\author{
Youfei Liu, Student Member, IEEE, and Felix F. Wu, Fellow, IEEE
}

\begin{abstract}
Nash equilibrium is usually used as the solution of generator's strategic bidding in electricity markets. The available literature has shown by simulation that multiple market equilibria or no pure equilibrium may be induced after the inclusion of network constraints (transmission constraints). This paper presents a systematical analysis for the impacts of network constraints on the market equilibrium in oligopolistic electricity markets. In our modeling, the independent system operator (ISO) dispatches generation and determines nodal prices via solving an optimal power flow (OPF); and the individual generator optimizes its submitted supply function with Nash-supply function equilibrium (SFE) strategy, after taking into account ISO's decision-making process on dispatched generations and nodal prices. The conditions for the existence of equilibrium are given, and it is shown that there may be multiple equilibria or no pure Nash equilibrium. The equilibrium, if it exists, will be at a point where generators pay no congestion charge.
\end{abstract}

Index Terms-Electricity markets, Nash equilibrium, optimal response curve, strategic bidding.

\section{INTRODUCTION}

$\mathbf{I}^{\prime}$ $\mathrm{N}$ recent years, competition has been introduced to the power industry in order to increase social welfare and to improve market efficiency. Usually, there is a spot market (day-ahead market) for short-term electricity transactions. In the sport market, market participants (generators and consumers) submit their next-day hourly generation or demand bids to an independent system operator (ISO). With submitted bids, the ISO clears the market to schedule generation and determine the market clearing price (MCP) [1]. Because at the current stage, the electricity market is more akin to an oligopoly, the individual market participant thereby has market power and can influence the MCP by its strategic bidding. Several papers have presented the empirical evidence for this issue [2], [3].

Roughly speaking, there are two important research issues related to the strategic bidding in electricity markets.

One is the study on how to build the optimal bidding strategy for the generator. The objective of optimal bidding is to achieve the maximum profit. Generally, the essence of the strategic bidding of a generator is to optimize its bid, based on its own production cost, its expectation about the others, and the market

Manuscript received May 2, 2006; revised August 26, 2006. Paper no. TPWRS-00271-2006.

Y. Liu is with the Dispatch Center, JiangXi Electric Power Company (JXEPC), NanChang, JiangXi, China (e-mail: youfei@eee.hku.hk).

F. F. Wu is with the Department of Electrical and Electronic Engineering, University of Hong Kong, Hong Kong.

Color versions of Figs. 2 and 15-17 are available online at http://ieeexplore. ieee.org.

Digital Object Identifier 10.1109/TPWRS.2006.889083 information. With the consideration of system demand uncertainties or generation bid uncertainties, the strategic bidding can also be formulated as a problem of maximizing the expected profit, which can be solved via various probabilistic/stochastic optimization methods, such as Markov decision processdiscrete dynamic programming [4], ordinal optimization [5], stochastic optimization with genetic algorithm, Monte Carlo simulation [6], Lagrangian relaxation, and stochastic dynamic programming [7].

On the other hand, optimal strategic bidding can be obtained via indirect methods, such as MCP-prediction-based methods and heuristic methods. MCP-prediction-based methods provide the bidding strategy for a price-taking generator. Usually, a price-taking generator first develops the method to forecast the MCP and then, with forecasted MCP, finds its optimal generation through self-unit scheduling. Many MCP prediction techniques are developed, such as ARIMA [8], cascaded neural network [9], and artificial intelligence [10]. Meanwhile, heuristic methods are proposed to develop the optimal bidding strategy via market learning, such as genetic algorithm evolution [11] and actor-critic learning algorithm [12].

Another important issue related to the strategic bidding is the analysis of market equilibrium, or the "steady" state of participant's strategic behavior. Game-theory-based methods are widely used to model strategic interactions between generators and thus search the Nash market equilibrium. In [13], a Nash game theory method is adopted to model and analyze the transactions in a Pool-Co market in a continuous strategy space with complete information. Paper [14] studies the impacts of potential coalitions on generators' bidding strategies by implementation of bids sensitivities. In [15] and [16], the supply function equilibrium (SFE) model is used to find the equilibrium by solving a set of coupled nonlinear equations. Authors in [17] and [18] find that the SFE model is an accurate descriptor of strategic agents. Paper [19] uses a three-step methodology to find multiperiod Nash equilibria, where an iterative simulation process is run to detect the market participants' bidding strategy, and then the output data from simulator is analyzed to pick out the Nash equilibria. It is well known that network constraints (such as transmission capacity limits) play important roles of system operation. Network constraints will induce market separation, where some generator can exercise its market power more easily and will be more likely to exploit these constraints. Thus, the study for the impacts of network constraints on the market equilibrium is strongly demanding. To our knowledge, most of the above reported works have ignored network constraints or just included them as a part of generator's bidding process; there is yet no effort to give a complete 
analytical study about the impacts of network constraints on the market equilibrium. Fortunately, some papers have shed some light on this issue by their simulations. The author in [20] develops an interesting multimarket spatial Cournot model in the form of a nonlinear programming optimization, and the simulation shows how generators may strategize to exercise local market power in the presence of transmission constraints. In [21], it is found by simulation that in a simple two-bus system, the pure strategy equilibrium is eliminated by the inclusion of network constraint. Furthermore, the authors simulate the same simple two-bus system and find that there exists a continuum of Nash equilibria when both consumer bidding and line constraint are considered [22]. In [23], in order to study the congestion influence on bidding strategies, the authors use a separate curtailment algorithm for congestion management and model generator's bidding as a three-level optimization problem. It is then demonstrated by simulation that in a three-node system, network constraint will cause no pure Nash equilibrium. In [24], a systematic procedure is developed for finding multiple-equlibria in a complicated system with network constraints, where an algorithm is developed to select the efficient regions for searching possible equilibria.

Given the above background, this paper presents analytical results about the impacts of network constraints on the market equilibrium in oligopolistic electricity markets. Generator bidding is modeled as a two-level optimization problem. In the first level, the ISO dispatches generation and determines nodal prices via solving an optimal power flow (OPF); while in the second level, after taking account of the ISO's decision-making process on dispatched generations and nodal prices, the generator optimizes its submitted linear supply function with Nash-supply function equilibrium (SFE) strategy. In our analysis, the whole decision space of generators is divided into different subregions. Within each subregion, the considered network constraint is either strictly binding or strictly slack. The Nash equilibrium is then located via searching the possible intersection of these optimal response curves within each region or on the boundary of these regions. First, a typical three-node system is used for investigation. It is shown that with the inclusion of a 'tight' network constraint, there may be a continuum of equilibria or no pure Nash equilibrium; and the market equilibrium, if it exists, can only be found on the constraint boundary, which implies that there is no nodal price difference or no congestion charge. The intuition that system congestion charge can be preempted by the generator strategic bidding has been pointed out by Oren in [25]. This fact may pose serious difficulties for proposals for congestion management protocols, such as in ERCOT, which rely on identifying a priori which lines are likely to be constrained.

This paper is arranged as follows. In Section II, the generator bidding in electricity markets is formulated as a two-level optimization problem. In Section III, using a typical network-constrained three-node system for investigation, the impacts of network constraints on the market equilibrium are analyzed. Discussions are presented in Section IV for generalizing the above conclusions to complicated systems. Numerical test results are presented in Section $\mathrm{V}$ with conclusions drawn in Section VI.

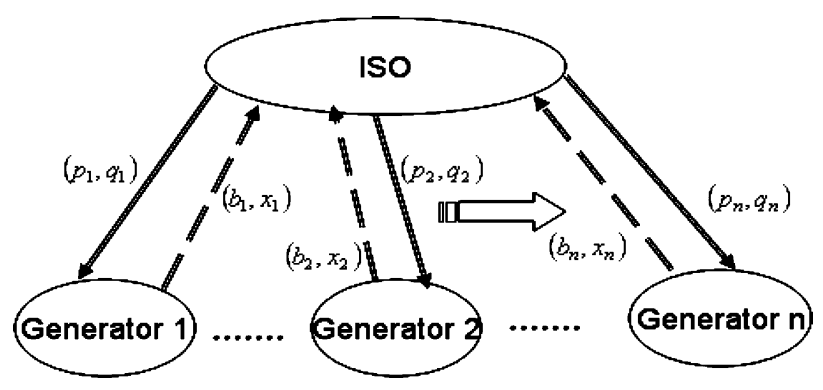

Fig. 1. Interaction between the ISO and generators.

\section{Modeling Generator Bidding as A Two-LeVel OPTIMIZATION PROBLEM}

\section{A. Basic Assumptions}

Usually, electricity demand can be described by some convex function of electricity price. Here we use a linear inverse demand function

$$
p=e-f D=e-f \sum_{i=1}^{n} q_{i}
$$

where $p$ is market price; $D$ is system demand; $n$ is the number of generators; and $e$ and $f$ are positive coefficients (fixed here). Usually as electrical power cannot be stored (in large amount), system supply and demand should be in instantaneous balance, i.e., $Q=\sum_{i=1}^{n} q_{i}=D$.

Each generator has a quadratic production cost function that takes the form

$$
C_{i}\left(q_{i}\right)=a_{i}+b_{i} q_{i}+\frac{1}{2} c_{i} q_{i}^{2}, \quad i=1, \ldots, n
$$

where $\left(a_{i}, b_{i}, c_{i}\right)$ are nonnegative coefficients.

Thus, the supply function (may not be the true marginal cost function) submitted by the generator to the ISO can take a linear form, generally given as

$$
p_{i}=b_{i}^{\prime}+x_{i} q_{i}
$$

In this paper, $b_{i}^{\prime}$ is replaced with the true cost parameter $b_{i}$ for a compact analysis. Thus, generator $i$ takes its supply function slope $x_{i}$ as the only decision variable for achieving the maximum profits.

\section{B. Two-Level Optimization Problem}

Similarly as in [21], the strategic bidding is modeled as a twolevel optimization problem. In the first level (shown in Fig. 1), the ISO solves an OPF with the aim of maximizing the social welfare (as revealed by the bids), i.e.,

$$
\begin{aligned}
& \underset{\left\{q_{1}, q_{2}, \ldots, q_{n}\right\}, D}{\max }\left[B(D)-\sum_{i=1}^{n} C_{i}\left(q_{i}\right)\right] \\
& \quad=\max _{\left\{q_{1}, q_{2}, \ldots, q_{n}\right\}, D}\left[\left(e D-\frac{1}{2} f D^{2}\right)-\sum_{i=1}^{n}\left(b_{i} q_{i}+\frac{1}{2} x_{i} q_{i}^{2}\right)\right] \\
& \text { S.T. } q_{1}+q_{2}+\cdots+q_{n}=D \\
& \quad g\left(q_{1}, q_{2}, \ldots, q_{n}, D\right) \leqslant 0 \\
& \quad q_{i}^{\min } \leqslant q_{i} \leqslant q_{i}^{\max }, \quad i=1, \ldots, n
\end{aligned}
$$


where $g\left(q_{1}, q_{2}, \ldots, q_{n}, D\right) \leqslant 0$ gives the network constraint (e.g., the transmission capacity limit).

The individual $q_{i}$ and corresponding nodal price $p_{i}$ $\left(p_{i}=b_{i}+x_{i} q_{i}\right)$ are determined from the optimization problem (4). It is clear that they are implicit functions of all submitted variables, defined as $q_{i}=\varphi_{i}\left(x_{1}, x_{2}, \ldots, x_{n}\right)$ and $p_{i}=\phi_{i}\left(x_{1}, x_{2}, \ldots, x_{n}\right)(i=1, \ldots, n)$.

Assume that the ISO's OPF solution process (4) is transparent to each player. Thus, in the second level (shown in Fig. 1), generator $i$ optimizes its supply function slope to maximize its profit, while taking into account the dispatched generation and nodal price determined in the first level by the ISO, i.e.,

$$
\begin{aligned}
\max _{x_{i}} \pi_{i} & =\max _{x_{i}}\left[p_{i} q_{i}-C_{i}\left(q_{i}\right)\right] \\
\text { S.T. } p_{i} & =\phi_{i}\left(x_{1}, x_{2}, \ldots, x_{n}\right) \\
q_{i} & =\varphi_{i}\left(x_{1}, x_{2}, \ldots, x_{n}\right) .
\end{aligned}
$$

Solving the problem (5), this yields generator $i$ 's optimal decision variable $x_{i}$, which is dependent on others' decision variables $x_{j}(j \neq i)$, given as

$$
x_{i}=\theta_{i}\left(x_{1}, x_{2}, \ldots, x_{i-1}, x_{i+1}, \ldots, x_{n}\right)=\theta_{i}\left(X_{-i}\right)
$$

where $X_{-i}$ is the vector of generator $i$ 's rivals decision variables $\left\{x_{j}\right\}(j \neq i)$. The function given by (6) is also known as optimal response function that describes generator's optimal response curve.

For the above two-level optimization problem, the interaction between the ISO and generators can be shown in Fig. 1.

\section{Concept of Market Equilibrium}

In the above game, the market decision variable is the supply function slope $\left\{x_{i}\right\}$; thus, the market equilibrium is also modeled by the variable $\left\{x_{i}\right\}$. Generally, the meaning of Nash equilibrium is that no one can get extra profit by unilaterally deviating from the equilibrium, i.e.,

$$
\pi_{i}\left(x_{i}^{*}, X_{-i}^{*}\right) \geqslant \pi_{i}\left(x_{i}, X_{-i}^{*}\right), \quad x_{i} \in \Psi_{i}, \quad i=1, \ldots, n
$$

where $\Psi_{i}$ is generator $i$ 's continuous decision space, given as $\Psi_{i}=\left\{x_{i} \mid x_{i} \in\left[0, x_{\max }\right]\right\}$ ( $x_{\max }$ is a big enough number). Because the definition of Nash equilibrium [e.g., (7)] is satisfied at the intersection point of optimal response curves (6), thus the market Nash equilibrium is given by the intersection of optimal response curves (6), i.e., $x_{i}^{*}=\theta_{i}\left(X_{-i}^{*}\right)(i=1, \ldots, n)$.

\section{CONTINUUM OF EQUILIBRIA}

To focus on how network constraints influence the market equilibrium, minimum and maximum generation constraints are neglected in this study. That is, constraints

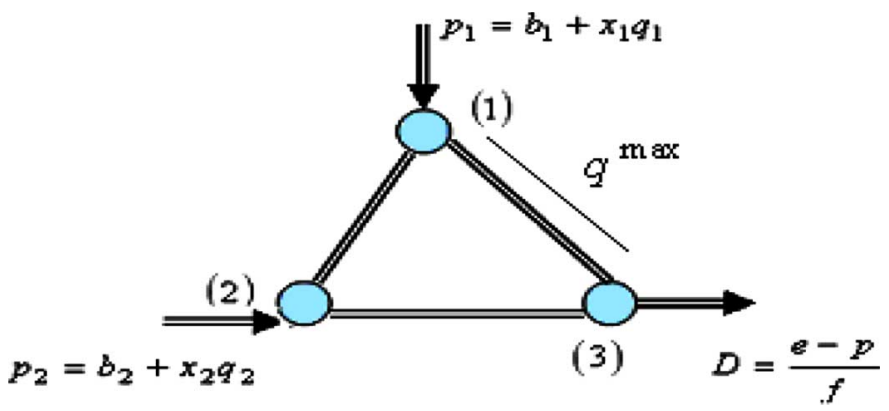

Fig. 2. Typical three-node system.

$q_{i}^{\min } \leqslant q_{i} \leqslant q_{i}^{\max }(i=1, \ldots, n)$ are eliminated in the OPF problem (4).

First, a typical network-constrained three-node system (e.g., two generating nodes and one demand node) is used for investigation, as shown in Fig. 2. It is assumed that there is the transmission limit on the link from node 1 to node 3 .

With a dc formulation, the network constraint can be modeled as (the demand side node is taken as the reference node)

$$
\beta_{1} q_{1}+\beta_{2} q_{2} \leqslant q^{\max }
$$

where $\beta_{1}$ and $\beta_{2}$ are the PTDF of generator 1 and generator 2 , respectively. Inequality (8) can be used to replace network constraint $g\left(q_{1}, q_{2}, \ldots, q_{n}, D\right) \leqslant 0$ in the OPF problem (4). The network constraint modeled by (8) has two statuses, either binding or slack. Via a constraint status decomposition (either binding or slack), the whole decision space $\Phi=\Psi_{1} \times \Psi_{2}$ can be divided into two regions $\Phi^{1}$ and $\Phi^{2}$, as shown in Figs. 4 and 10. Within region $\Phi^{1}$, the system is not congested, $\left(\Phi^{1}=\right.$ $\left\{\left(x_{1}, x_{2}\right) \mid \mu=0\right.$ or $\left.\left.\left(q^{\max }-\beta_{1} q_{1}+\beta_{2} q_{2}\right)>0\right\}\right)$. Within region $\Phi^{2}$, the system is congested, i.e., $\Phi^{2}=\left\{\left(x_{1}, x_{2}\right) \mid \mu>=\right.$ 0 or $\left.\left(q^{\max }-\beta_{1} q_{1}+\beta_{2} q_{2}\right)=0\right\}$. That is, the constraint (8) is strictly slack in region $\Phi^{1}$, and it is strictly binding in region $\Phi^{2}$. Nevertheless, the market equilibrium is still searched through the possible intersection of optimal response curves within these inner regions or on the boundary between these regions.

\section{A. Optimal Response Curve in Congestion-Off Region $\Phi^{1}$}

First, the situation without network constraints is explored. In the OPF problem (4), the constraint $g\left(q_{1}, q_{2}, \ldots, q_{n}, D\right) \leqslant$ 0 is thus eliminated. With (1)-(5), it is not difficult to derive generator $i$ 's profit function and obtain its first-order derivative w.r.t. its decision variable $x_{i}$, given in (9), shown at the bottom of the next page.From the first-order condition for optimality

$$
\frac{\partial \pi_{i}}{\partial x_{i}}=\frac{\left(e-b_{i}+f \sum_{j=1, j \neq i}^{n} \frac{b_{j}-b_{i}}{x_{j}}\right)^{2}\left[f+\left(c_{i}-x_{i}\right)\left(1+f \sum_{j=1, j \neq i}^{n} \frac{1}{x_{j}}\right)\right]}{\left[x_{i}\left(1+f \sum_{j=1, j \neq i}^{n} \frac{1}{x_{j}}\right)+f\right]^{3}}
$$




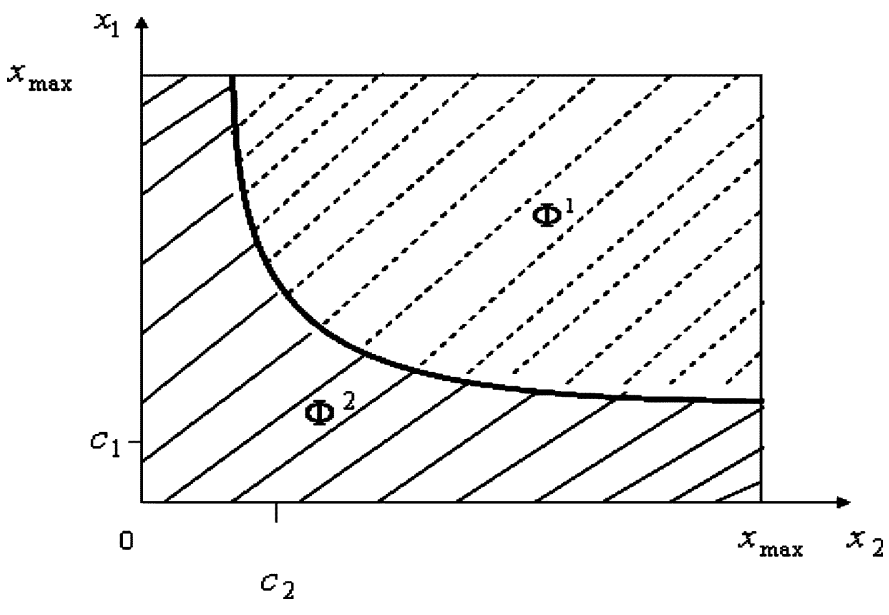

Fig. 3. Decision space of generators.

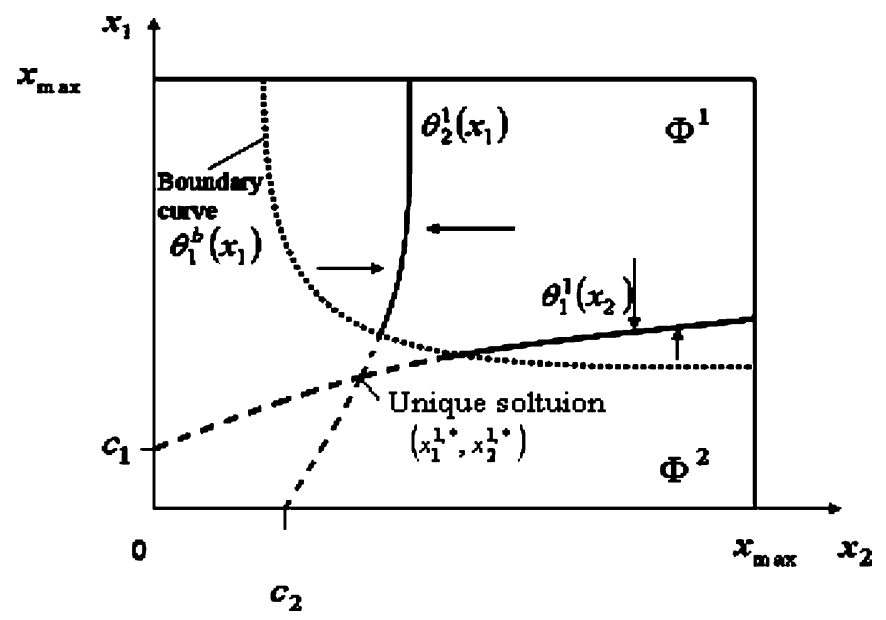

Fig. 4. Optimal response curves in the congestion-off region.

$\left(\partial \pi_{i} / \partial x_{i}=0\right)$, the generator's optimal response curve is given as

$$
\left\{\begin{array}{l}
x_{1}=\theta_{1}^{1}\left(x_{2}\right)=c_{1}+\frac{f}{1+f \frac{1}{x_{2}}} \\
x_{2}=\theta_{2}^{1}\left(x_{1}\right)=c_{2}+\frac{f}{1+f \frac{1}{x_{1}}} .
\end{array}\right.
$$

Detailed derivation of the above formulations can be found in [26]. In [26], it is shown that the coupled equation system (10) has the unique solution $\left(x_{1}^{1, *}, x_{2}^{1, *}\right)$, as shown in Fig. 4. In Fig. 4, these vertical arrows show the trajectory of generator 1 's decision variable $x_{1}$, while these horizontal arrows show the trajectory of generator 2's decision variable $x_{2}$. It is not surprising that all of them move toward the respective optimal response curve.

As aforementioned, the whole decision space is divided into two regions. The boundary curve between the congestion-off region $\Phi^{1}$ and congestion-on region $\Phi^{2}$ is characterized by

$$
\left\{\begin{array}{l}
b_{1}+x_{1} q_{1}=b_{2}+x_{2} q_{2}=e-f\left(q_{1}+q_{2}\right) \\
\beta_{1} q_{1}+\beta_{2} q_{2}=q^{\max }
\end{array}\right.
$$

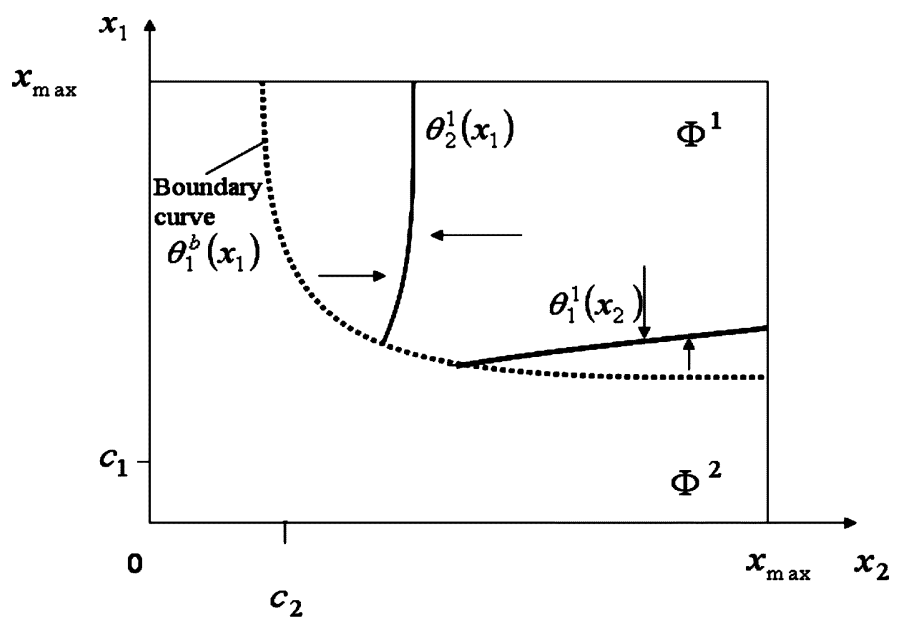

Fig. 5. Valid optimal response curves in the congestion-off region.

With some mathematical manipulation, formulation (11) will yield

$$
\begin{array}{r}
q^{\max } x_{1} x_{2}+\left[f q^{\max }-\left(e-b_{2}\right) \beta_{2}\right] x_{1}+\left[f q^{\max }-\left(e-b_{1}\right) \beta_{1}\right] x_{2} \\
+f\left(b_{1}-b_{2}\right)\left(\beta_{1}-\beta_{2}\right)=0 .
\end{array}
$$

Thus, on the boundary, we can also define the optimal response function, given as

$$
\left\{\begin{array}{l}
x_{1}=\theta_{1}^{b}\left(x_{2}\right)=-\frac{f\left(b_{1}-b_{2}\right)\left(\beta_{1}-\beta_{2}\right)+\left[f q^{\max }-\left(e-b_{1}\right) \beta_{1}\right] x_{2}}{q^{\max } x_{2}+\left[f q^{\max }-\left(e-b_{2}\right) \beta_{2}\right]} \\
x_{2}=\theta_{2}^{b}\left(x_{1}\right)=-\frac{f\left(b_{1}-b_{2}\right)\left(\beta_{1}-\beta_{2}\right)+\left[f q^{\max }-\left(e-b_{2}\right) \beta_{2}\right] x_{1}}{q^{\max } x_{1}+\left[f q^{\max }-\left(e-b_{1}\right) \beta_{1}\right]} .
\end{array}\right.
$$

When there is the transmission limit on the link from node 1 to node 3 , both $\beta_{1}$ and $\beta_{2}$ are positive, and the whole decision space $\Phi$ is divided into two subregions (i.e., a congestion-off region $\Phi^{1}$ and a congestion-on region $\Phi^{2}$ ) by the boundary curve (12), which is shown in Fig. 3.

Note that optimal response curves (10) can intersect in the region $\Phi^{1}$ or in the region $\Phi^{2}$. When these optimal response curves still intersect within the region $\Phi^{1}$, the market equilibrium is given as the unique solution of coupled equation system (10), i.e., $\left(x_{1}^{1, *}, x_{2}^{1, *}\right)\left(\in \Phi^{1}\right)$ is the equilibrium. If so, it is said that network constraint is too slack, and it cannot affect the market equilibrium. In this paper, the impacts of network constraints on the equilibrium are the focus. Thus, it is assumed that the considered network constraint is "tight," and optimal response curves [given by (10)] do not intersect within the region $\Phi^{1}$, i.e., the unique solution of (10) is located in the region $\Phi^{2}$ $\left(\left(x_{1}^{1, *}, x_{2}^{1, *}\right) \in \Phi^{2}\right)$, as shown in Fig. 4 .

These dotted lines represent the invalid optimal response curves as they are located in an incompatible region (i.e., they are unconstrained optimal response curves located in the constrained region). The valid optimal responses in the congestion-off region are then shown in Fig. 5.

Hence, within the region $\Phi^{1}$, there is no equilibrium as there is no intersection of optimal response curves. Note that $\left(x_{1}^{1, *}, x_{2}^{1, *}\right)$ (located in the region $\left.\Phi^{2}\right)$ is no longer the market equilibrium. 
Within the region $\Phi^{2}$, the individual generator has another kind of optimal response curve, discussed in the following section.

\section{B. Generators' Optimal Response Curve in the Region $\Phi^{2}$}

Within the region $\Phi^{2}$, the considered network constraint is strictly binding, i.e., an equality constraint $\beta_{1} q_{1}+\beta_{2} q_{2}=q^{\max }$. Thus, the ISO's OPF problem is reformulated as

$$
\begin{aligned}
& \max _{q_{1}, q_{2}, D}\left[\left(e D-\frac{1}{2} f D^{2}\right)-\left(b_{1} q_{1}+\frac{1}{2} x_{1} q_{1}^{2}\right)\right. \\
& \left.\quad-\left(b_{2} q_{2}+\frac{1}{2} x_{2} q_{2}^{2}\right)\right] \\
& \text { S.T. } q_{1}+q_{2}=D \\
& \quad \beta_{1} q_{1}+\beta_{2} q_{2}=q^{\max } .
\end{aligned}
$$

With the help of Lagrangian multipliers to associate the constraints into the objective function, the above problem can be formulated as a Lagrangian function, given as

$$
\begin{aligned}
H= & \left(e D-\frac{1}{2} f D^{2}\right)-\left(b_{1} q_{1}+\frac{1}{2} x_{1} q_{1}^{2}\right) \\
& -\left(b_{2} q_{2}+\frac{1}{2} x_{2} q_{2}^{2}\right)+\lambda\left(q_{1}+q_{2}-D\right) \\
& +\mu\left(q^{\max }-\beta_{1} q_{1}-\beta_{2} q_{2}\right)
\end{aligned}
$$

where the Lagrangian multiplier $\mu$ gives the shadow price of network constraint, and the Lagrangian multiplier $\lambda$ gives the marginal cost for market balancing. For the above system, $\lambda$ is also the market price in the demand side.

Solving (15), this yields the scheduled generation

$$
\left\{\begin{aligned}
q_{1} & =\varphi_{1}\left(x_{1}, x_{2}\right) \\
& =\frac{\left(e-b_{1}\right) \beta_{2}^{2}-\left(e-b_{2}\right) \beta_{1} \beta_{2}+f\left(\beta_{1}-\beta_{2}\right) q^{\max }+x_{2} \beta_{1} q^{\max }}{f\left(\beta_{1}^{2}+\beta_{2}^{2}-2 \beta_{1} \beta_{2}\right)+x_{1} \beta_{2}^{2}+x_{2} \beta_{1}^{2}} \\
q_{2} & =\varphi_{2}\left(x_{1}, x_{2}\right) \\
& =\frac{\left(e-b_{2}\right) \beta_{1}^{2}-\left(e-b_{1}\right) \beta_{1} \beta_{2}+f\left(\beta_{2}-\beta_{1}\right) q^{\max }+x_{1} \beta_{2} q^{\max }}{f\left(\beta_{1}^{2}+\beta_{2}^{2}-2 \beta_{1} \beta_{2}\right)+x_{1} \beta_{2}^{2}+x_{2} \beta_{1}^{2}} .
\end{aligned}\right.
$$

The first-order derivative of generator's profit function w.r.t. its decision variable is given in (17) at the bottom of the page. Generators' optimal response functions are given as [from the firstorder conditions for optimality $\left(\partial \pi_{1} / \partial x_{1}=0\right.$ and $\partial \pi_{2} / \partial x_{2}=$ $0)]$

$$
\left\{\begin{array}{l}
x_{1}=\theta_{1}^{2}\left(x_{2}\right)=\frac{f\left(\beta_{1}^{2}+\beta_{2}^{2}-2 \beta_{1} \beta_{2}\right)+x_{2} \beta_{1}^{2}}{\beta_{2}^{2}}+c_{1} \\
x_{2}=\theta_{2}^{2}\left(x_{1}\right)=\frac{f\left(\beta_{1}^{2}+\beta_{2}^{2}-2 \beta_{1} \beta_{2}\right)+x_{1} \beta_{2}^{2}}{\beta_{1}^{2}}+c_{2} .
\end{array}\right.
$$

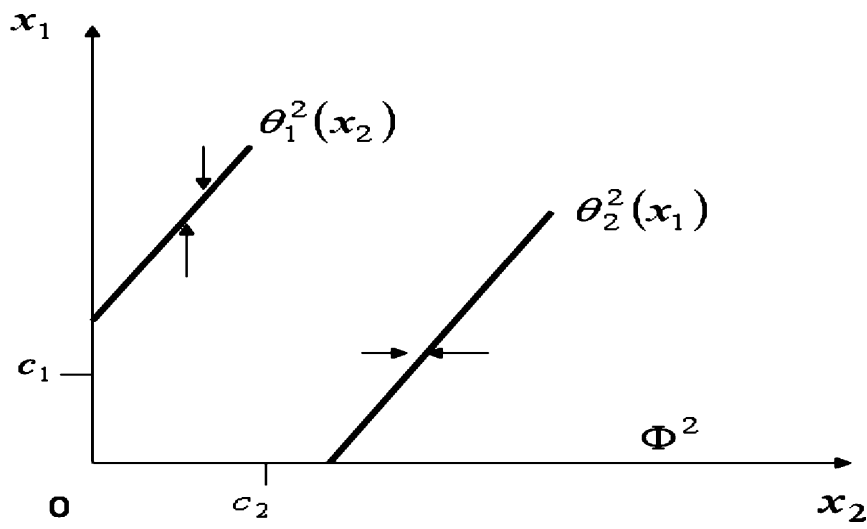

Fig. 6. Optimal response curves in the congestion-off region.

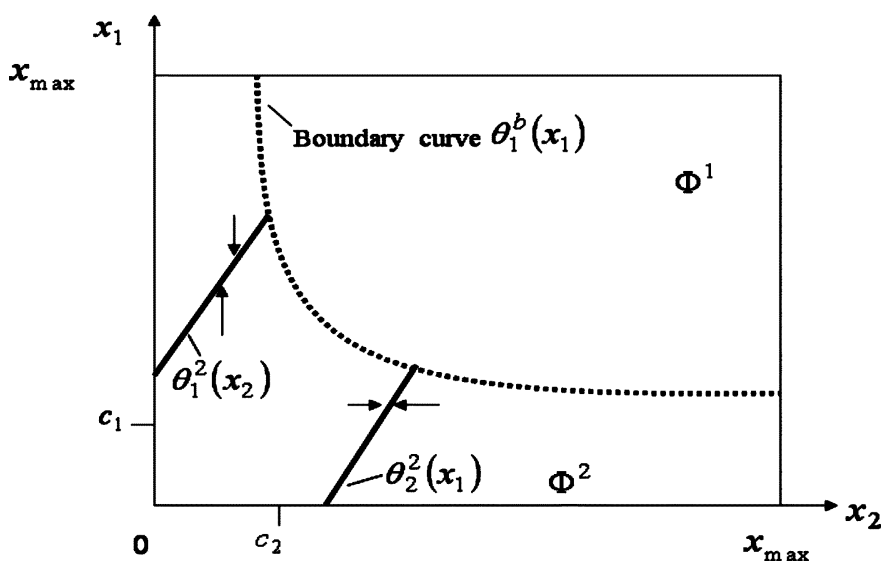

Fig. 7. Valid optimal response curves in the congestion-on region.

Optimal response curves in the congestion-on region $\Phi^{2}$ are shown in Fig. 6.

Considering the whole decision space, the valid optimal response curves in the congestion-on region $\Phi^{2}$ are then given by Fig. 7.

It is very easy to check that these optimal response curves within the region $\Phi^{2}$, given by (18), are parallel, and thus, there is no intersection within the region $\Phi^{2}$. That is to say that there is no equilibrium within the region $\Phi^{2}$.

Integrating Figs. 5 and 7, the complete optimal response curves in the whole decision space are obtained, as shown in Fig. 8.

It is to see that there is a section of the boundary curve that attracts all the movements of $x_{1}$ and $x_{2}$, i.e., there is a continuum

$$
\left\{\begin{array}{l}
\frac{\partial \pi_{1}}{\partial x_{1}}=\left[\left(e-b_{1}\right) \beta_{2}^{2}-\left(e-b_{2}\right) \beta_{1} \beta_{2}+f\left(\beta_{1}-\beta_{2}\right) q^{\max }+x_{2} \beta_{1} q^{\max }\right]^{2} \\
\quad \times \frac{\left[f\left(\beta_{1}^{2}+\beta_{2}^{2}-2 \beta_{1} \beta_{2}\right)-x_{1} \beta_{2}^{2}+x_{2} \beta_{1}^{2}+c_{1} \beta_{2}^{2}\right]}{\left[f\left(\beta_{1}^{2}+\beta_{2}^{2}-2 \beta_{1} \beta_{2}\right)+x_{1} \beta_{2}^{2}+x_{2} \beta_{1}^{2}\right]^{3}} \\
\frac{\partial \pi_{2}}{\partial x_{2}}=\left[\left(e-b_{2}\right) \beta_{1}^{2}-\left(e-b_{1}\right) \beta_{1} \beta_{2}+f\left(\beta_{2}-\beta_{1}\right) q^{\max }+x_{1} \beta_{2} q^{\max }\right]^{2} \\
\quad \times \frac{\left[f\left(\beta_{1}^{2}+\beta_{2}^{2}-2 \beta_{1} \beta_{2}\right)-x_{2} \beta_{1}^{2}+x_{1} \beta_{2}^{2}+c_{2} \beta_{1}^{2}\right]}{\left[f\left(\beta_{1}^{2}+\beta_{2}^{2}-2 \beta_{1} \beta_{2}\right)+x_{1} \beta_{2}^{2}+x_{2} \beta_{1}^{2}\right]^{3}}
\end{array}\right.
$$




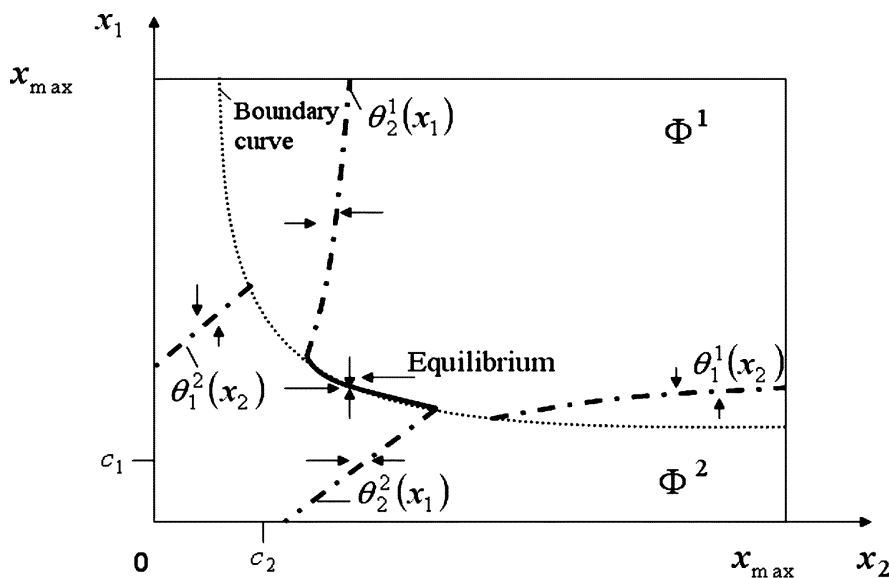

Fig. 8. Complete optimal response curves.

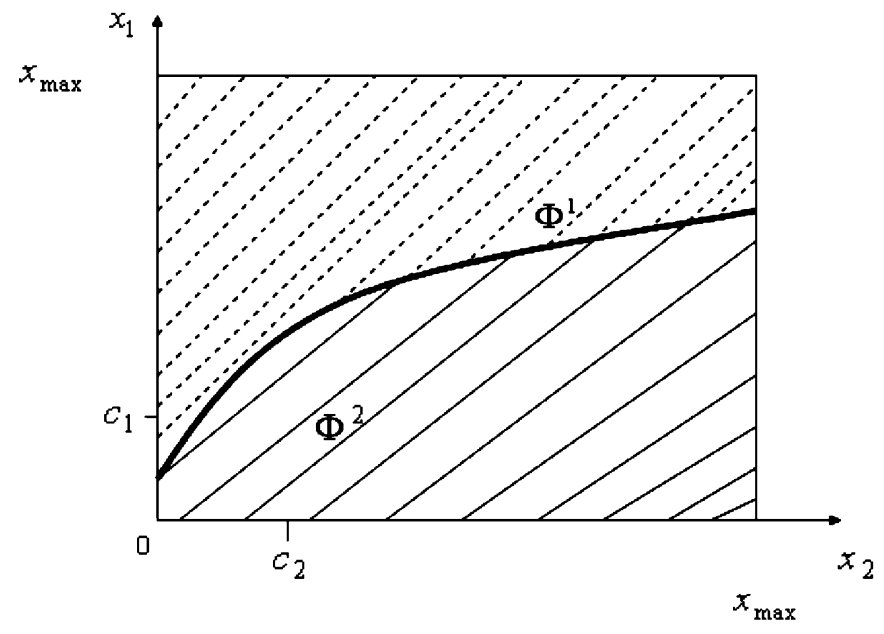

Fig. 9. Decision space of generators.

of equilibria on the boundary, shown in Fig. 8 with black solid line.

On the other hand, if the network constraint exists on the link between node 2 and node 3 , it is obvious that it is a symmetrical situation with above one, and it can be expected that the similar results can be obtained.

\section{No PURE EQUILIBRIUM}

However, if there is a network constraint on the link between node 1 and node 2 (the system is shown in Fig. 2), a completely different result is obtained. Without loss of generality, it is assumed that this network constraint may be binding in the direction from node 1 to node 2 (for generator 1 has a relative larger absolute value of PTDF in this line, i.e., $\left.\left|\beta_{1}\right|>\left|\beta_{2}\right|\right)$. Using a dc load flow formulation, demand side node (node 3 ) as the reference node, the network constraint still can be modeled by (8). However, now $\beta_{2}$ is negative, which means that on the link between node 1 and node 2 , the power flow attributed by generator 2 is the counter flow to the one attributed by generator 1. It is still clear that this network constraint divides generators' decision space into two regions, as shown in Fig. 9. The only difference is that we have a different shape of the boundary curve.

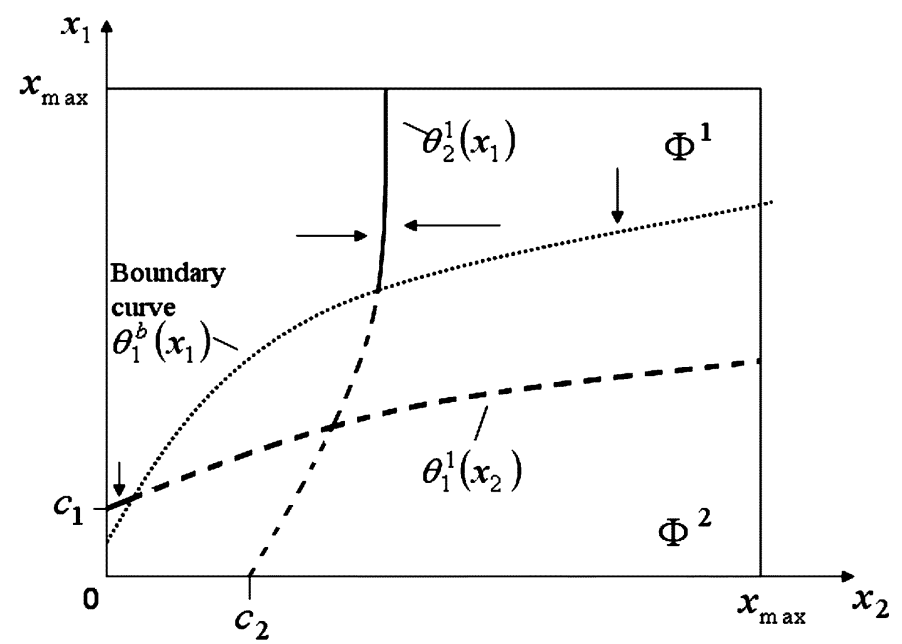

Fig. 10. Optimal response curves in congestion-off region.

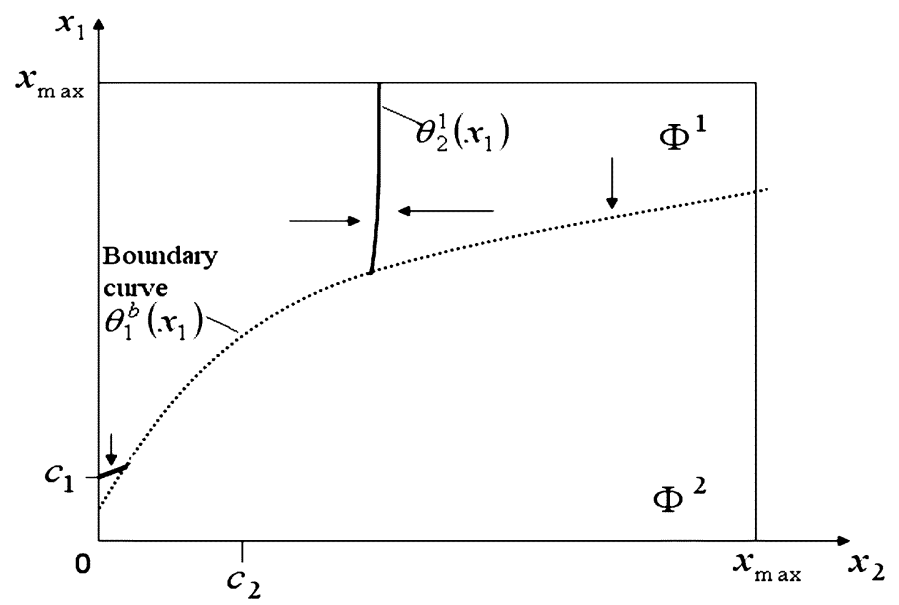

Fig. 11. Valid optimal response curves in congestion-off region.

When $x_{2}=0$, the interception of boundary curve on axis $x_{1}$ is given as $x_{1}=-\left(f\left(b_{1}-b_{2}\right)\left(\beta_{1}-\beta_{2}\right)\right) /$ $\left(\left[f q^{\max }-\left(e-b_{2}\right) \beta_{2}\right]\right)$ [from (12)]. This point can be below point $x_{1}=c_{1}$ or not. However, this trivial difference will not alter our analytical results. Here, it is assumed that this interception point is below point $x_{1}=c_{1}$.

\section{A. Optimal Response Curve in Congestion-Off Region $\Phi^{1}$}

In the congestion-off region $\Phi^{1}$, generators' optimal response curves are still modeled by (10). Fig. 10 shows these optimal response curves.

These dotted lines represent the invalid optimal response curves as they are located in the wrong region. After eliminating these invalid optimal response curves, the valid response curves in the congestion-off region are then given by Fig. 11.

\section{B. Optimal Response Curve in the Congestion-On Region $\Phi^{2}$}

In the congestion-on region $\Phi^{2}$, generators' optimal response curves are still modeled by (18). Fig. 12 shows these optimal response curves.

These dotted lines represent the invalid optimal response curves as they are located in an incompatible region. After 


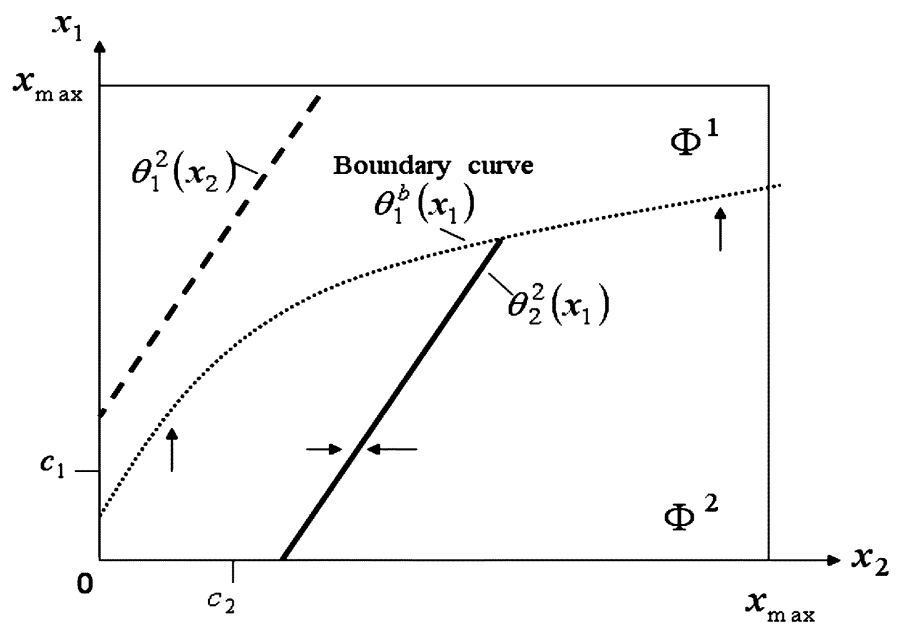

Fig. 12. Optimal response curves in congestion-on region.

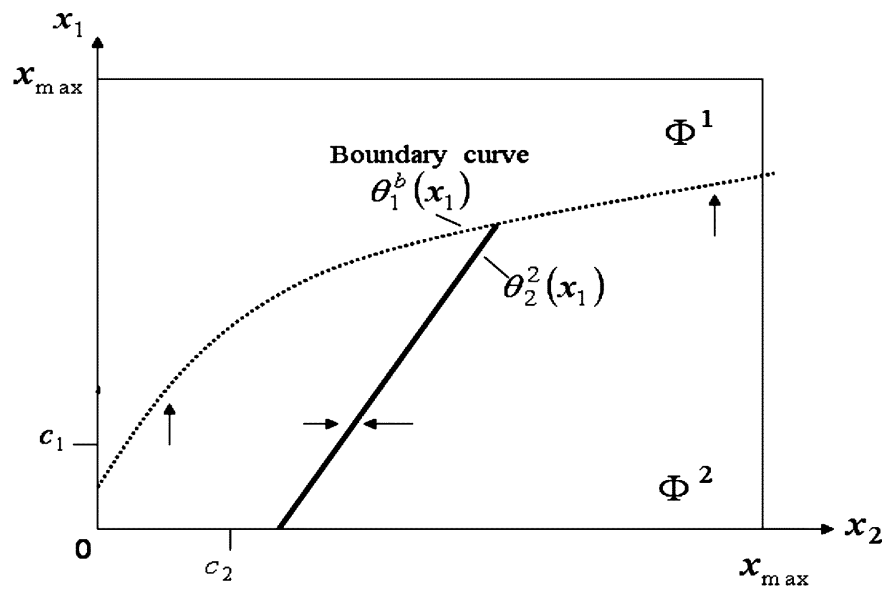

Fig. 13. Valid optimal response curves in congestion-off region.

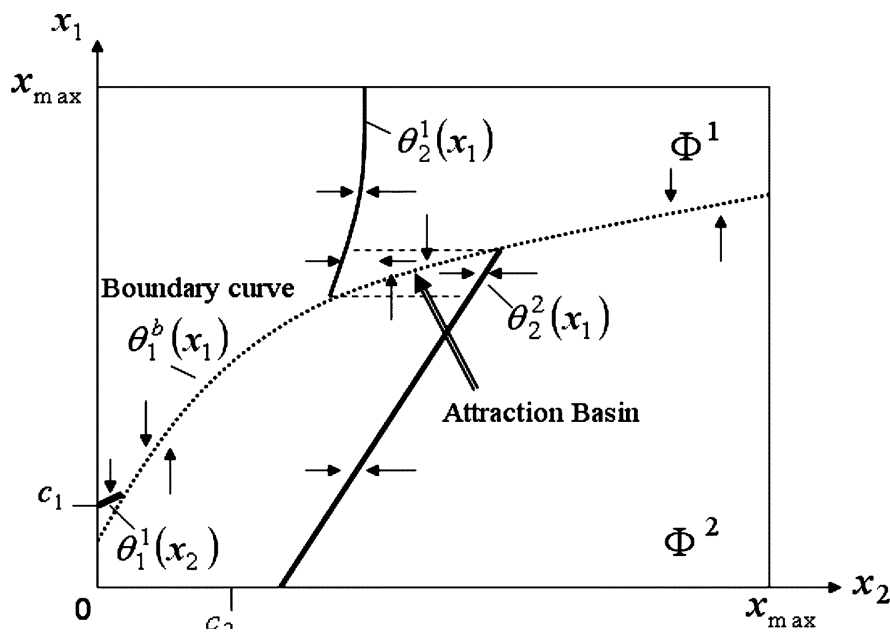

Fig. 14. Complete optimal response curves.

eliminating these invalid optimal response curves, the valid response curves in the congestion-on region are then given by Fig. 13.

Integrating Figs. 11 and 13, the complete optimal response curves in the whole decision space are obtained, as shown in Fig. 14.

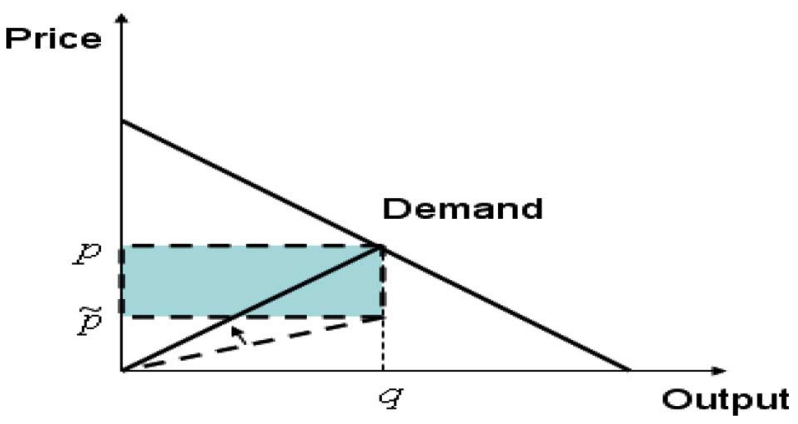

Fig. 15. Illustration for preempting the congestion charge.

With the same argument, the market equilibrium is located as the intersection of optimal responses. From Fig. 14, it can be concluded that there is no pure market equilibrium as there is no intersection of optimal response curves. However, it is clear that there is an attraction basin that attracts all the movements from outside. Inside this basin, generator 2's decision variable $x_{2}$ has two different movement directions, either moving left to $\theta_{2}^{1}\left(x_{1}\right)$, which is located in region $\Phi^{1}$, or moving right to $\theta_{2}^{2}\left(x_{1}\right)$, which is located in region $\Phi^{2}$. Thus, it is expected that there may be some complex phenomena happening inside the attraction basin, such as limit cycle or chaos. Such issues are left for future work, due to the limited space.

Oren in [25] also analyzes a similar case. However, he assumes that in node 1, there is an equivalent monopoly generator (or implicit collusion by many generators); and in node 2, there are perfect competitive generators (no market power, or equivalently there is one generator with $x_{2}=c_{2}$ ). Thus, in [25], the only equilibrium is located at the point $\left(\theta_{1}^{b}\left(c_{2}\right), c_{2}\right)$, and also there is no congestion charge.

\section{FURTHER DISCUSSION}

\section{A. Case 1: Network Constraint on the Line Between Node 1 (Generator 1) and Node 3 (Demand Side)}

Our analysis in Section III shows that the equilibrium can only be found on the boundary implies that in equilibrium, there is no nodal price difference (the corresponding Lagrangian multiplier $\mu$ or the shadow price of network constraint is zero). To explain such a fact, look at Fig. 15.

As shown in Fig. 15, if the considered network constraint is binding and thus some generators' outputs are limited, there will be different nodal prices. Based on these nodal price differences, the ISO will collect congestion charges, which are given as the shadow area $((p-\tilde{p}) q)$. After observing that the ISO can collect congestion charges, all generators can mark up their bids to release system congestion and take up a part of initial "congestion charges" as their extra profits (in this situation, all generator's PTDF on the "congested" line are all positive). Such strategic behaviors actually push the network constraint from strictly binding to the slack. On the other hand, when the network constraint is strictly slack, all generators will lower their bids to increase their generations for more profits. In this situation, generators' strategic behaviors actually push the constraint to be binding. These strategic behaviors are shown in Section III. Thus, in any situation, all generators' strategic behaviors are 
consistent, either driving the constraint from strictly slack to binding or driving the constraint from strictly binding to slack. These consistent strategic behaviors thereby induce the market equilibrium only to be located on the boundary where the network constraint is just binding, and they results in no congestion charge. Usually on the constraint boundary, there is a continuum of equilibria.

The above analysis can be generalized to complicated systems. We conjecture that as long as in a complicated system, the inclusion of tight network constraints (transmission capacity limits) induces consistent strategic behaviors for all generators, there still could be a continuum of equilibria on the network constraint boundary (where the network constraint is just binding), and there is no system congestion charge.

\section{B. Case 2: Network Constraint on the Line Between Node 1}

\section{(Generator 1) and Node 2 (Generator 2)}

In this situation, there is no pure Nash equilibrium, as analyzed in Section IV.

The key difference between case 1 and case 2 is that in case 2 , the directions of power flow from generator 1 and generator 2 are just opposite ( $\beta_{1}$ is positive and $\beta_{2}$ is negative). Thus, the counter flow attributed by generator 2 will cancel a part of power flow attributed by generator 1 and thereby mitigate the system congestion in the link from node 1 to node 2 . Check nodal prices from the OPF (15), given as

$$
\left\{\begin{array}{l}
p_{1}=\lambda-\mu \beta_{1} \\
p_{2}=\lambda+\mu\left(-\beta_{2}\right) \\
p_{3}=\lambda .
\end{array}\right.
$$

If the studied network constraint is binding, the Lagrangian multiplier $\mu$ is positive and the market price in node 2 will be greater than the price in node $1\left(p_{2}>p_{1}\right)$. For generator 2 , when the constraint is strictly binding, it can lower its bid to increase its scheduled generation $q_{2}$ and still obtains more profits, although the system congestion is mitigated (i.e., although $p_{2}$ is decreased, the increased $q_{2}$ makes generator 2 still profitable). At the same time, it can also mark up its bid to decrease its scheduled generation $q_{2}$; thus, it aggravates system congestion, increases nodal price $p_{2}$, and still obtains more profits (i.e., although $q_{2}$ is decreased, the increased $p_{2}$ makes generator 2 still beneficial). For generator 1, when the network constraint is strictly binding, it will always mark up its bid to release the congestion for more profits. Therefore, when network constraint is binding, strategic behaviors from generator 1 and generator 2 are inconsistent, and such inconsistent strategic behaviors actually induce no pure Nash equilibrium.

The above analysis can also be generalized to complicated systems. We conjecture that in a complicated system, if the introduction of tight network constraints (transmission capacity limits) induces inconsistent strategic behaviors from generators, there still could be no pure Nash equilibrium.

It should be pointed out that it is generally impossible to give some thorough analytical results for a large complicated system. Nevertheless, we can adopt some numerical technique to verify the above conjectures, such as the method in [28].

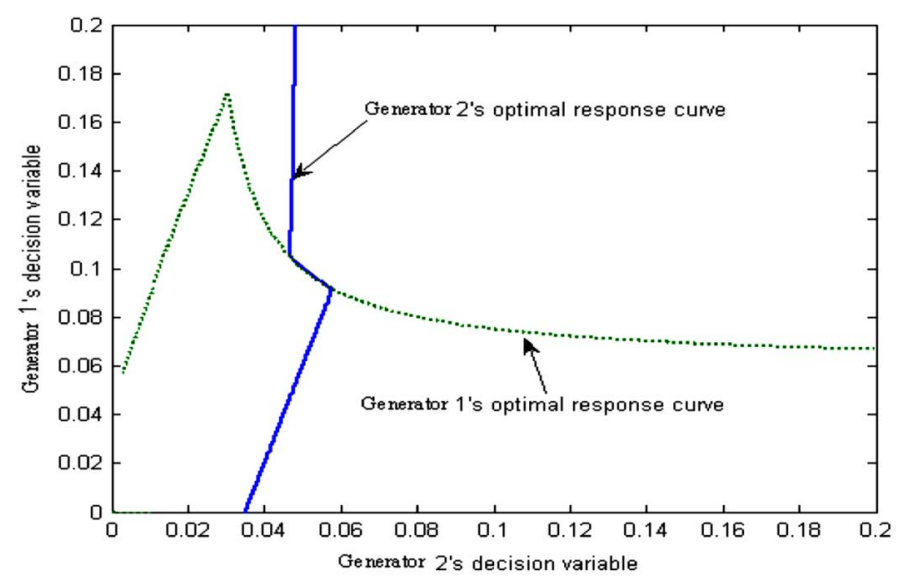

Fig. 16. Optimal response curves with network constraint from node 1 to node 3.

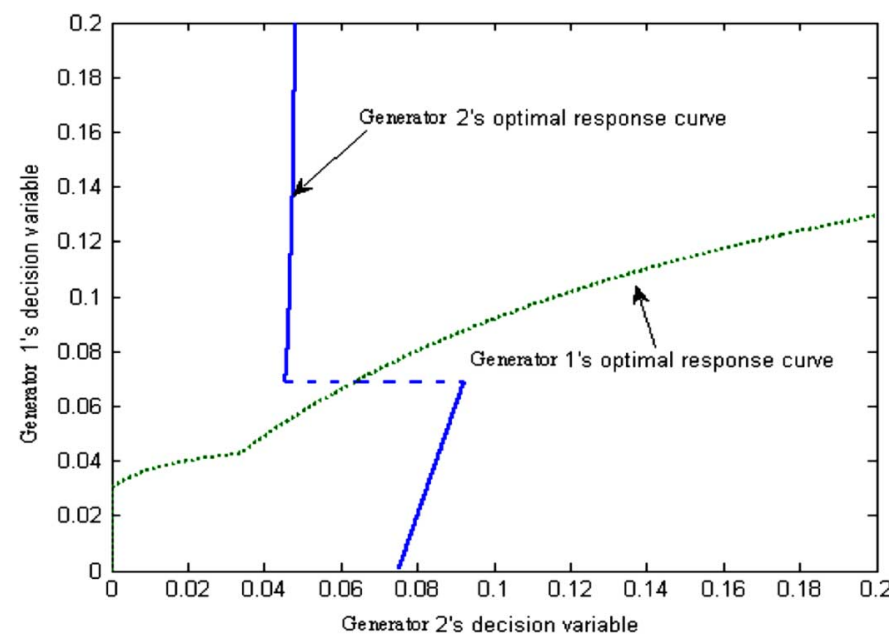

Fig. 17. Optimal response curves with network constraint from node 1 to node 2.

\section{NUMERICAL RESULTS}

To validate our analytical conclusions, numerical results in a three-node system (shown in Fig. 2) are given in this section.

The system inverse demand function is given as

$$
p=50-0.02 D=5-0.02\left(q_{1}+q_{2}\right) .
$$

The generator's quadratic cost function is given as

$$
C(q)=10+2 q+\frac{1}{2} 0.03 q^{2}
$$

\section{Test 1: Network Constraint on the Line From Node 1 to Node 3}

The network constraint is given as $0.5 q_{1}+0.25 q_{2} \leqslant 300$. A numerical technique is used to obtain optimal response curve. For example, given a $x_{2}$ in each step, $x_{1}$ is varied in its decision range to find the corresponding $x_{1}$ that gives generator 1 the maximum profit. Repeating the above calculation step by step, this yields generator 1's optimal response curve. Similarly, 
generator 2's optimal response curve can be obtained. Finally, generators' optimal response curves are shown in Fig. 16.

As analyzed in Section III, there is a continuum of equilibria, which are given as the intersections of these two optimal response curves.

\section{Test 2: Network Constraint on the Line From Node 1 to Node 2}

In this situation, network constraint is given as $0.5 q_{1}-$ $0.25 q_{2} \leqslant 100$. The similar numerical technique as in test 1 is used to obtain generators' optimal response curves. Generators' optimal response curves are shown in Fig. 17.

Also as analyzed in Section IV, it is easy to see that there is no pure strategy equilibrium as the obtained optimal response curves have no intersection (the dotted line of generator 1's optimal response curve means the jump/discontinuity of generator 1 's decision variable).

\section{CONCLUSION}

This paper presents a systematic analysis about the impacts of network constraints (transmission constraints) on electricity market equilibrium. In our investigation, the generator bidding is modeled as a two-level optimization problem. In the first level, the ISO dispatches generations and determines nodal prices via solving an OPF. While, in the second level, the individual generator adopts Nash-SFE strategy and bids a linear supply function to maximize its profit after taking into account the ISO's decision-making process on the dispatched generation and nodal prices. Using a three-node system for investigation, it is shown that there may be a continuum of equilibrium or no pure Nash equilibrium. The equilibrium, if it exists, can only be found on the constraint boundary, which implies that there is no nodal price difference or no congestion charge. Later on, the above conclusions are generalized to complicated systems. It is argued that in complicated systems, if the binding network constraints induce consistent generators" "bidding around the constraint" behaviors, then there will still be a continuum of equilibira. On the other hand, if the binding network constraints induce some inconsistent generators' bidding behavior, then there may be no pure Nash equilibrium.

It is known that some existing electricity markets propose to use system congestion charges as a major reason of system transmission expansion and planning. This paper has shown that generators' strategic behavior can significantly alter system congestion charges and maintain prices in excess of the competitive level. This could potentially increase the difficulty of planning the transmission system for an independent operator.

Our future work will be to study the system with many imposed network constraints and many competitive generators.

\section{ACKNOWLEDGMENT}

The authors would like to thank S. Li for her help as well as the three anonymous referees and the editor for their helpful suggestions, discussions, and comments.

\section{REFERENCES}

[1] M. Shahidehpour and M. Almoush, Restructured Electrical Power Systems: Operation, Trading, and Volatility. New York: Marcel Dekker, 2001.
[2] S. Borenstein and J. Bushnell, "Market power in electricity markets: Beyond concentration measures," Energy J., vol. 20, no. 4, pp. 65-88, 1999.

[3] S. Borenstein, J. Bushnell, E. Kahn, and S. Stoft, "Market power in California electricity markets," Util. Pol., vol. 5, no. 3-4, pp. 219-236, 1995.

[4] H. Song, C.-C. Liu, J. Lawarrée, and R. W. Dahlgren, "Optimal electricity supply bidding by Markov decision process," IEEE Trans. Power Syst., vol. 15, no. 2, pp. 618-624, May 2000.

[5] X. Guan, Y.-C. Ho, and F. Lai, "An ordinal optimization based bidding strategy for electric power suppliers in the daily energy market," IEEE Trans. Power Syst., vol. 16, no. 4, pp. 788-797, Nov. 2001.

[6] F. Wen and A. K. David, "Optimal bidding strategies and modeling of imperfect information among competitive generators," IEEE Trans. Power Syst., vol. 16, no. 1, pp. 15-21, Feb. 2001.

[7] E. Ni, P. B. Luh, and S. Rourke, "Optimal integrated generation bidding and scheduling with risk management under a deregulated power market," IEEE Trans. Power Syst., vol. 19, no. 1, pp. 600-609, Feb. 2004.

[8] J. Contreras, R. Espinola, F. J. Nogales, and A. J. Conejo, "ARIMA models to predict next-day electricity prices," IEEE Trans. Power Syst., vol. 18, no. 3, pp. 1014-1020, Aug. 2003.

[9] L. Zhang, P. B. Luh, and K. Kasiviswanathan, "Energy clearing price prediction and confidence interval estimation with cascaded neural networks," IEEE Trans. Power Syst., vol. 18, no. 3, pp. 991-05, Aug. 2003.

[10] Y. Y. Hong and C. Y. Hsiao, "Locational marginal price forecasting in deregulated electricity markets using artificial intelligence," Proc. Inst. Elect. Eng.,Gen., Transm., Distrib., vol. 149, no. 5, pp. 621-626, Sep. 2002.

[11] C. W. Jr. Richter and G. B. Sheble, "Genetic algorithm evolution of utility bidding strategies for the competitive marketplace," IEEE Trans. Power Syst., vol. 13, no. 1, pp. 256-261, Feb. 1998.

[12] G. R. Gajjar, S. A. Khaparde, P. Nagaraju, and S. A. Soman, "Application of actor-critic learning algorithm for optimal bidding problem of a Genco," IEEE Trans. Power Syst., vol. 18, no. 1, pp. 11-18, Feb. 2003.

[13] J. B. Park, B. H. Kim, J. H. Kim, M. H. Jung, and J. K. Park, "A continuous strategy game for power transactions analysis in competitive electricity markets," IEEE Trans. Power Syst., vol. 16, no. 4, pp. 847-855, Nov. 2001.

[14] Y. He and Y.H. Song, "The study of the impacts of potential coalitions on bidding strategies of Gencos," IEEE Trans. Power Syst., vol. 18, no. 3, pp. 1086-1093, Aug. 2001.

[15] A. Rudkevich, M. Duckworth, and R. Rosen, "Modeling electricity pricing in a deregulated generation industry: The potential for oligopoly pricing in a Poolco," Energy J., vol. 19, no. 3, pp. 19-48, 1998.

[16] R. Baldick, R. Grant, and E. Kahn, "Linear supply function equilibrium: Generalizations, applications, and limitations," University of California Energy Institute POWER Paper PWP-078, Aug. 2000. [Online]. Available: http://www.ucei.berkeley.edu/PDF/pwp078.pdf.

[17] S. Hortacau and S. Puller, "Testing strategic models of firm behavior in restructured electricity markets: A case study of ERCOT," Center for the Study of Energy Markets, 2003, Paper CSEMWP-125.

[18] C. J. Day, B. F. Hobbs, and J.-S. Pang, "Oligopolistic competition in power networks: A conjectured supply function approach," IEEE Trans. Power Syst., vol. 17, no. 3, pp. 597-607, Aug. 2002.

[19] S. DelaTorre, J. Contreras, and A. J. Conejo, "Finding multiperiod Nash equilibria in pool-based electricity markets," IEEE Trans. Power Syst., vol. 19, no. 1, pp. 643-651, Feb. 2004.

[20] D. Chattopadhyay, "Multi-commodity spatial Cournot model for generator bidding strategy," IEEE Trans. Power Syst., vol. 19, no. 1, pp. 267-275, Feb. 2004.

[21] J. D. Weber and T. J. Overbye, "A two-level optimization problem for analysis of market bidding strategies," in Proc. IEEE Power Eng. Soc. Summer Meeting, Edmonton, AB, Canada, 1999, vol. 2, pp. 682-687.

[22] J. D. Weber and T. J. Overbye, "An individual welfare maximization algorithm for electricity markets," IEEE Trans. Power Syst., vol. 17, no. 3, pp. 590-596, Aug. 2002.

[23] T. S. Peng and K. Tomsovic, "Congestion influence on bidding strategies in an electricity market," IEEE Trans. Power Syst., vol. 18, no. 3, pp. 1054-1061, Aug. 2003.

[24] P. F. Correia, T. J. Overbye, and I. A. Hiskens, "Searching for noncooperative equilibria in centralized electricity markets," IEEE Trans. Power Syst., vol. 18, no. 4, pp. 1417-1424, Nov. 2003.

[25] S. S. Oren, "Economic inefficiency of passive transmission rights in congested electricity systems with competitive generation," Energy J., vol. 18 , no. 1, pp. 63-83, 1997. 
[26] Y. F. Liu, Y. X. Ni, and F. F. Wu, "Existence, uniqueness, stability of linear supply function equilibrium in electricity markets," in Proc. IEEE Power Eng. Soc. General Meeting, Denver, CO, Jul. 2004, pp. 249-254.

[27] A. J. Wood and B. F. Wollenberg, Power Generation, Operation, and Control, 2nd ed. New York: Wiley, 1996.

[28] L. B. Cunningham, R. Baldick, and M. L. Baughman, "An empirical study of applied game theory: Transmission constrained Cournot behavior," IEEE Trans. Power Syst., vol. 17, no. 1, pp. 166-172, Feb. 2002.

Youfei Liu (S'04) received the B.S. and M.S. degrees in control theory and control engineering from University of Science and Technology of China (USTC), Hefei, China, in 1999 and 2002, respectively, and the doctoral degree from the Electrical and Electronic Engineering Department, Hong Kong University, Hong Kong, in 2006.

Currently, he is with the Dispatch Center, JiangXi Electric Power Company, NanChang, JiangXi, China. His research interests are power industry deregulation and power system analysis, stability, and control.
Felix F. Wu (F'89) received the Ph.D. degree from University of California at Berkeley (UCB).

$\mathrm{He}$ is now a Chair Professor of the Electrical and Electronic Engineering Department, Hong Kong University, Hong Kong. Prior to that, he was a Professor and Vice-Chair of Department of Electrical Engineering and Computer Science at UCB. His research interests are electric energy industry restructuring, power system investment planning, design of modern control centers, and distributed processing. 